\title{
Outcome for biliary atresia patients treated at a low-volume centre
}

Kristin Bjørnland ${ }^{1,2}$, Maren Hinna ${ }^{2}$, Gunnar Aksnes ${ }^{1}$, Kjetil Juul Stensrud ${ }^{1}$, Kjetil Ertresvåg ${ }^{1}$, Anniken Bjørnstad-Østensen ${ }^{2,3}$, Truls Sanengen ${ }^{3}$, Pål-Dag Line ${ }^{4,2}$, Ingegerd Aagenæs ${ }^{5}$, Lars Aabakken $^{6,2}$, Ragnhild Emblem ${ }^{1,2}$, Runar Almaas ${ }^{3,7}$

${ }^{1,2}$ Department of Gastro and Paediatric Surgery, Oslo University Hospital

${ }^{2}$ University of Oslo

${ }^{3}$ Department of Paediatrics, Oslo University Hospital

${ }^{4,2}$ Department of Transplantation Medicine, Oslo University Hospital

${ }^{5}$ Department of Radiology, Oslo University Hospital

${ }^{6,2}$ Department of Medicine, Oslo University Hospital

${ }^{7}$ Department of Pediatric Research, Oslo University Hospital

Running title: Outcome for biliary atresia

Corresponding author: Kristin Bjørnland. Dep. of Gastro and Paediatric Surgery, Oslo

University Hospital Rikshospitalet. Postboks 4950 Nydalen. 0424 Oslo, Norway 


\section{ABSTRACT}

Objectives: The importance of case load for treatment of extrahepatic biliary atresia (BA) is debated. The aim of this study was to register results of BA treatment in a small volume centre.

Methods: Retrospective chart review study of patients with BA treated from 2000 to 2017. The institutional review board approved the study.

Results: 45 babies were identified of which $42(93 \%)$ are alive. 41 patients had a Kasai portoenterostomy (PE), two a hepaticojejunostomy and two a primary liver transplantation. The age at PE/hepaticojejunostomy was median 63 (4-145 days). Seven surgeons performed the operations, and the median duration of the diagnostic work-up was 8 (3-24) days. Clearance of jaundice was achieved in 23/43 (53\%) babies, and 3- and 5-year native liver survival was $47 \%$ and $40 \%$ respectively. Clearance of jaundice post PE/hepaticojejunostomy was a strong predictor of native liver survival (adjusted OR: 0.027; 95\%; $\mathrm{P}=0.009$ ). Plasma level of conjugated bilirubin at time of referral was also a significant predictor of native liver survival (adjusted OR: 1.053; $\mathrm{P}=0.017$ ).

Conclusion: A small volume centre may achieve satisfactory results for BA patients. The study has, however, identified factors that may further improve results; earlier referral, optimizing diagnostic work-up, and establishing one dedicated surgical team.

Abbreviations: BA: Biliary atresia; PE: Portoenterostomy

Key-words: Biliary atresia, Kasai, portoenterostomy, liver transplantation, hepaticojejunostomy, clearance of jaundice, hepaticojejunostomy 


\section{Introduction}

Extrahepatic biliary atresia (BA) is an inflammatory condition obliterating the biliary ducts in neonates. This rare disease is of unknown aetiology, affecting 1:14000-1:20000 live births in Europe (1). Both the intra and extrahepatic ducts are affected by progressive destruction leading to cholestasis, fibrosis and cirrhosis. Around one fifth of BA patients have other congenital malformations of which the biliary atresia splenic malformation syndrome is most common. Left untreated, BA is fatal within the first years of life. Kasai portoenterostomy (PE) is usually the first line treatment. After a successful PE, the child's plasma bilirubin level normalizes, and extended native liver survival may be anticipated. In the best centres, clearance of jaundice is reported in $75 \%$ of the cases $(2,3)$. If the Kasai PE is unsuccessful, liver failure develops, and the child will need a liver transplantation.

Over the past two decades the relationship between case load of complicated surgical procedures and outcome has been extensively debated. Centralization of BA surgery has resulted in significantly improved outcome in several countries, for instance the United Kingdom and Finland $(2,4)$. A similar trend has not been shown in series from France and Canada $(5,6)$. It is therefore important for all centres treating BA patients to evaluate own data, both in terms of clearance of jaundice, postoperative complications and native liver survival. If the results are unsatisfactory, the organization of treatment for BA should be discussed. Thus, the aim of this study was to register outcome of BA treatment in a centre treating few BA patients annually.

\section{Patients and methods}

In Norway, patients with suspected BA are presently admitted to Oslo University Hospital Rikshospitalet (OUS) where paediatric hepatologists coordinate the diagnostic work-up. Ultrasonography and hepatobiliary scintigraphy are always performed. Additional imaging modalities of the biliary system (endoscopic retrograde cholangiopancreaticography (ERCP), percutaneous cholecystocholangiography (PCC), cholangiogram performed by laparoscopy/laparotomy) are selected based on individual assessment.

\section{Study design and definitions}

This is a retrospective observational chart review study of all patients, except one refugee with incomplete medical data, with confirmed BA treated at OUS from January 2000 to February 2017. One Norwegian patient has been successfully operated at another hospital in the study period, but data from this patient is not included in this series. Some of the data from patients operated between January 2005 and June 2016 have recently presented in a multicentre Nordic study (7). Clearance of jaundice was defined as postoperative plasma bilirubin level $\leq 20 \mu \mathrm{mol} / \mathrm{l}$ within six months after PE. Postoperative complications were classified according to the Clavien-Dino classification system (8). Due to the retrospective nature of the study, Grade $\mathrm{O}$ (no complication) and Grade 1 complications were summarized.

For the statistical analyses and in the discussion, hepaticojejunostomies and PEs are grouped together as PEs. 


\section{Ethics}

The study was approved by the institutional review board (2016/16847), and written informed consent was obtained from all parents.

\section{Statistical analysis}

Numerical data are presented as median [min-max] because they did not fit normal distribution. Categorical data are presented as frequencies and percentages. Mann-Whitney U-test was used to compare continuous variables and Chi-square to compare categorical data. In the multivariate analysis, adjusted odd ratio (OR) and $95 \%$ confidence intervals (CI) are presented. A P-value less than 5\% was considered statistically significant. All analyses were performed using IBM SPSS Statistics for Windows, Version 24.0. Armonk, NY: IBM Corp. Released 2016.

\section{Results}

\section{Patient demographics}

Forty-five babies, 29 girls (64\%), were diagnosed with BA during the study period. Weight at admittance was median 4600 (1935-5980) grams, and total and conjugated plasma bilirubin were median 162 (72-352) and $110(25-216) \mu \mathrm{mol} / \mathrm{l}$, respectively. The median age at last follow-up was 86 (4-194) months. Three patients died at the age of 18, 26 and 51 months respectively; all had been operated with a Kasai procedure. One of the patients died of causes unrelated to BA with normal liver function at the age of 26 months, whereas the two other patients died 15 and 46 months after liver transplantation.

Data on associated malformations were unobtainable in six patients. In those where such information was available, 74\% (29/39) did not have any associated malformations. Details of the various malformations are summarized in table 1.

\section{Diagnostic work-up}

Standard blood tests, hepatobiliary scintigraphy and ultrasound were performed in all patients. A median of two (1-5) ultrasonographic examinations were performed at OUS. ERCP was performed in 27 children, $\mathrm{PCC}$ in 15, and ten babies were investigated with MRCP. The median time from admittance to OUS-R to PE was 8 (3-24) days.

\section{Operative details and postoperative complications}

A PE was performed in 41 babies, two received a hepaticojejunostomy, and two children had a primary liver transplantation due to advanced liver disease. Seven pediatric surgeons performed the PE, and the operative time was median 158 (106-225) minutes. There was no peri- or 90-day postoperative mortality. Additional peri- and postoperative data are listed in 
table 2.The median age at PE or hepaticojejunostomy was 63 (4-145) days. There was no significant difference in age at PE between the first 21 patients, operated between 2000 and 2008, (median 65 (14-145) days) and the last 22 patients (median 58 (4-104) days), ( $\mathrm{P}=0.899)$. From 2005, all patients received postoperative steroids in tapering doses for six weeks, ursodeoxycholic acid (URSO) for at least one year and cholangitis prophylaxis (trimethoprimsulpha) for three months.

\section{Clearance of jaundice and native liver survival}

Clearance of jaundice after PE was achieved in 23 babies (53\%). To examine whether clearance rate improved during the long study period, the patients were divided into two groups. From 2000-2008, the clearance rate among the 21 patients operated in this period was $41 \%$ as opposed to $67 \%$ among the 22 patients operated between 2009 and 2017 ( $\mathrm{P}=0.091$ ). Seven patients had a PE later than 90 days of age. In these patients, the clearance rate was $29 \%$. The preoperative level of plasma conjugated bilirubin was significantly lower in the group of patients with clearance of jaundice after PE (table 3) 22 patients (median age 56 months (4-194)) are alive with their native liver, but one is currently awaiting liver transplantation. Three- and five year native liver survival was $47 \%$ and $40 \%$ respectively. Clearance of jaundice post PE was a strong predictor of native liver survival. Thus, $87 \%$ (20/23) who experienced clearance of jaundice had their native liver at follow-up as opposed to $15 \%(3 / 20)$ of those who did not normalize plasma bilirubin postoperatively $(\mathrm{P}<0.001)$.

Two patients received a primary liver transplantation at the age of five months. Both are alive. Twenty have been transplanted due to progressive liver dysfunction post PE, and 90\% $(18 / 20)$ are alive. Liver transplantation was performed at median seven (5-62) months.

\section{Discussion}

We have shown that a small volume center may achieve results for BA patients comparable to those reported from centers treating a larger volume of BA patients. The clearance rate of jaundice in this series was $54 \%$, and $87 \%$ of those with cleared jaundice, had their native liver median 4.5 years later. There was no peri-operative mortality and few post-operative complications. Furthermore, overall survival was $93 \%$.

The clearance rate following PE may partly be viewed as a reflection of the surgery (9). The highest reported clearance rates after PE are around $75 \%$ in recent papers $(2,3)$. After centralization of BA surgery in England and Wales to three supra-regional centers, clearance of jaundice was achieved in 55\% after PE (10). A recent survey from the Nordic countries, including some of the present patients, showed that centers treating $>3$ yearly achieved the best results (11). Although several data show better outcome after PE in specialized centers, not all studies come to the same conclusion $(5,6)$. In our current series, the overall clearance rate was 53\%. Notably, the patients operated in the second half of this 16 year period, had a clearance rate of $67 \%$. Thus, recently we observed a clearance rate after PE comparable to those reported in recent literature. 
$\mathrm{PE}$ is a rarely performed and a complicated operative procedure. In many centers only one or two surgeons perform the operations for BA $(3,9,12)$. Even though the results of centralization to larger centers have been addressed, no particular studies have examined how the experience of the individual surgeon influences outcome of PE. The $43 \mathrm{PE}$ in this series were performed by seven different surgeons, and if a trainee performed the PE, an experienced consultant was always present. Our numbers are too small to assess the impact of individual surgeon experience. Even so, it should be considered whether these infrequent operations should be performed by a dedicated team in order to further improve the results.

$\mathrm{PE}$ is a major operation and as such a certain number of early and late complications is expected. Surprisingly, very few papers report on early complications which may include bleeding, bile leaks, anastomotic leaks, small bowel obstruction, and internal hernias (13). In this series, less than a fifth experienced any complication. Because of the retrospective nature of this study, some minor complications may have been missed. Still, taking into account that some of these children had advanced liver disease and that major surgery was performed, the complication rate is considered acceptable.

In addition to the clearance rate of jaundice, native liver survival after PE is an important outcome measure following PE (14). Native liver survival reflects both the status of the liver at PE, the result of the PE and the medical management after PE. To ensure this, a multidisciplinary team ensuring appropriate nutrition and vitamin supplements is essential, as well as appropriate clinical follow-up. Although we have a small series with limited follow-up time, $87 \%$ of patients who cleared their jaundice, had their native liver at a median of 4.5 years postoperatively. When comparing long-time native liver survival, our results are not as good as the best results published $(2,10)$. The low number of patients makes it difficult to compare results and evaluate improvement over time, and hopefully long-time native liver survival will increase in patients operated now since clearance rate of bilirubin in patients operated in the last period has increased compared to previous years.

A lot of research has focused on predictors of PE outcome (15). We found that a lower level of conjugated plasma bilirubin immediately prior to PE, predicted a higher rate of both clearance of jaundice and native liver survival. To the best of our knowledge, this has not been reported before. This interesting finding should be studied further in a larger patient population.

The diagnostic work-up for BA vary between centers. Hepatobiliary scintigraphy and ultrasound are important and valuable screening modalities for obstructive biliary disorders. If multiple ultrasonographic features are evaluated, BA can be distinguished from other causes of conjugated hyperbilirubinemia in $98 \%$ of infants (16). A very detailed assessment of the gallbladder length, shape and wall regularity was not performed in all patients in the present series. It is possible that a more systematic ultrasonographic examination might have demonstrated findings highly suggestive of BA in some patients which could have been referred to surgery without any further investigations. In our center, percutaneous liver biopsies are not undertaken to diagnose BA because interpretation of biopsies may be difficult and takes time (7). Therefore, either ERCP or PCC have been performed. In all of the patients 
where BA was suspected by ERCP, BA was confirmed (results not shown). We have previously reported a very low complication rate after ERCP in infants at our hospital (17).

In this series the median time from admittance to the pediatric gastroenterologist at Oslo university hospital to PE, was 8 days. This included all preoperative investigations. The time used from admittance at a specialist center to surgery, is rarely reported in the literature. In the Netherlands, median time from referral to surgery was 12 days (18). It also took median 12 days from referral to a pediatric gastroenterologist to PE for Scottish infants operated at one of the three supra-regional centers in England (19). Thus, approximately a week from referral to PE, is well within the time spent for diagnostic work-up in other European centers. Still, it may be possible to further reduce this time. One might consider omitting ERCP/PCC in children where the ultrasonographic findings are clearly suggestive of BA. Furthermore, an operative cholangiography may be done laparoscopically, and this procedure is probably not much more invasive than an ERCP/PCC. By doing a laparoscopic cholangiography, the PE may be done directly if findings are suggestive of BA.

How age at PE affects outcome is still debatable, although there seems to be an agreement that early surgery is associated with better outcome $(9,20)$. The median age at PE in the present series was 63 days, and the patients operated in the latter half of the period was one week younger than in the former half. In Finland, between 2005 and 2009, median age at PE was 57 days, which is similar to our series (2). Among 424 patients operated in England, median age at PE was 54 days (10). Even though the median age at PE in recent years is comparable with those from other European countries, there is still a significant number of our patients that are referred late. $20 \%$ of our patients were operated after 90 days of age, and half of these patients were born after 2008. Thus, it seems clear that primary health care providers in Norway need more information about BA and the importance of early referral for icteric infants.

The strength of this study is that all the patients in the country, except one successfully operated in another hospital some years ago, diagnosed with BA in the time period are included. The main limit is that the series is small, making detailed statistical analyses inappropriate or underpowered. Furthermore, for some of the patients operated in the early years of the study, some data were not possible to retrieve.

\section{Conclusion}

This retrospective review of results of BA treatment in a small volume centre, shows that outcomes for the patients are comparable to those from larger centers. Even so, the study has identified factors that may further improve results; these include one dedicated surgical team, optimizing diagnostic work-up after referral and better education of primary health care providers to ensure earlier referral. 
Table and figure legends:

Table 1. Associated malformations in patients diagnosed with biliary atresia between 2000 and 2017. Data on malformations were available in 39/45 patients. Some patients had more than one malformation.

Table 2. Postoperative complications in patients operated with Kasai portoenterostomy or hepaticojejunostomy for biliary atresia classified according to the Clavien-Dindo classification system (8). Data were able to retrieve in 34 patients. Some patients had more than one complication.

Table 3. Factors that may influence the clearance rate of jaundice after portoenteostomy (PE). Median (min, max) values are given. *At admittance at Oslo university hospital for suspected biliary atresia 


\section{References}

1. Hartley JL, Davenport M, Kelly DA. Biliary atresia. Lancet. 2009;374:1704-1713.

2. Lampela H, Ritvanen A, Kosola $\mathrm{S}$, et al. National centralization of biliary atresia care to an assigned multidisciplinary team provides high-quality outcomes. Scand I Gastroenterol. 2012;47:99-107.

3. Kvist N, Davenport M. Thirty-four years' experience with biliary atresia in Denmark: a single center study. Eur J Pediatr Surg. 2011;21:224-228.

4. Davenport M, De Ville de Goyet J, Stringer MD, et al. Seamless management of biliary atresia in England and Wales (1999-2002). Lancet. 2004;363:1354-1357.

5. Serinet $\mathrm{MO}$, Broue $\mathrm{P}$, Jacquemin $\mathrm{E}$, et al. Management of patients with biliary atresia in France: results of a decentralized policy 1986-2002. Hepatology. 2006;44:75-84.

6. Schreiber RA, Barker CC, Roberts EA, et al. Biliary atresia in Canada: the effect of centre caseload experience on outcome. J Pediatr Gastroenterol Nutr. 2010;51:61-5.

7. Pakarinen MP, Johansen LS, Svensson JF, et al. Outcomes of biliary atresia in the Nordic countries - a multicenter study of 158 patients during 2005-2016. J Pediatr Surg. 2017, sept E-pub ahead of print.

8. Dindo D, Demartines N, Clavien PA. Classification of surgical complications: a new proposal with evaluation in a cohort of 6336 patients and results of a survey. Ann Surg. 2004;240:205213.

9. Davenport $\mathrm{M}$, Caponcelli E, Livesey $\mathrm{E}$, et al. Surgical outcome in biliary atresia: etiology affects the influence of age at surgery. Ann Surg. 2008;247:694-698.

10. Davenport $\mathrm{M}$, Ong E, Sharif K, et al. Biliary atresia in England and Wales: results of centralization and new benchmark. J Pediatr Surg. 2011;46:1689-1694.

11. Pakarinen MP, Pakkasjarvi N, Merras-Salmio L, et al. Intestinal rehabilitation of infantile onset very short bowel syndrome. J Pediatr Surg. 2015;50:289-292.

12. Sibanda N, Lewsey JD, van der Meulen JH, Stringer MD. Continuous monitoring tools for pediatric surgical outcomes: an example using biliary atresia. J Pediatr Surg. 2007;42:19191925.

13. Grisotti G, Cowles RA. Complications in pediatric hepatobiliary surgery. Semin Pediatr Surg. 2016;25:388-394.

14. Davenport M. Biliary atresia: clinical aspects. Semin Pediatr Surg. 2012;21:175-184.

15. Lakshminarayanan B, Davenport M. Biliary atresia: A comprehensive review. J Autoimmun. 2016;73:1-9.

16. Humphrey TM, Stringer MD. Biliary atresia: US diagnosis. Radiology. 2007;244:845-851.

17. Aabakken L, Aagenaes I, Sanengen T, et al. Utility of ERCP in neonatal and infant cholestasis. J Laparoendosc Adv Surg Tech A. 2009;19:431-436.

18. de Vries W, de Langen ZJ, Groen H, et al. Biliary atresia in the Netherlands: outcome of patients diagnosed between 1987 and 2008. J Pediatr. 2012;160:638-644.

19. Tayler R, Barclay AR, Rogers P, et al. Scottish outcomes for extra hepatic biliary atresia postrationalisation of services. Arch Dis Child. 2013;98:381-383.

20. Sokol RJ. Swiss outcomes in biliary atresia: are there lessons to be learned? J Pediatr Gastroenterol Nutr. 2008;46:238-240. 
Table 1

\begin{tabular}{|l|l|}
\hline Type of malformation & $\mathbf{N}(\%)$ \\
\hline No malformation & $29(74)$ \\
\hline Biliary atresia splenic malformation syndrome & $5(13)$ \\
Preduodenal portal vein & $1(3)$ \\
Situs inversus & $1(3)$ \\
\hline Cardiac malformation & $5(13)$ \\
\hline Anorectal malformation & $1(3)$ \\
\hline Esophageal atresia & $1(3)$ \\
\hline Intestinal malrotation & $1(3)$ \\
\hline Ileal atresia & $1(3)$ \\
\hline Cerebral anomalies & $1(3)$ \\
\hline
\end{tabular}

Table 2

\begin{tabular}{|l|c|}
\hline Grade 0 and 1 & $29(85 \%)$ \\
\hline Grade 2 & $4(12 \%)$ \\
Cholangitis & $2(4 \%)$ \\
Septicaecemia & $2(4 \%)$ \\
\hline Grade 3 & $2(4 \%)$ \\
Bile leakage needing drainage & $1(2 \%)$ \\
Colon perforation & $1(2 \%)$ \\
\hline
\end{tabular}

Table 3

\begin{tabular}{|l|c|c|c|}
\cline { 2 - 4 } \multicolumn{1}{c|}{} & Clearance & No clearance & P-value \\
\hline Age at PE (days) & $58(4-101)$ & $65(13-145)$ & 0.543 \\
\hline Total plasma bilirubin* $(\mu \mathrm{mol} / \mathrm{l})$ & $154(72-256)$ & $190(118-352)$ & 0.120 \\
\hline Conjugated plasma bilirubin* $\mu \mathrm{mol} / \mathrm{l}$ & $98(25-181)$ & $135(74-216)$ & 0.009 \\
\hline
\end{tabular}

